\title{
Response to Shah et al: Using high-resolution variant frequencies empowers clinical genome interpretation and enables investigation of genetic architecture
}

Nicola Whiffin, ${ }^{1,2,3}$ Angharad Roberts, ${ }^{1,2}$ Eric Minikel, ${ }^{4,5}$ Zach Zappala, ${ }^{4,5}$ Roddy Walsh, ${ }^{1,2}$ Anne H O'Donnell-Luria, ${ }^{4,5}$ Konrad J Karczewski, ${ }^{4,5}$ Steven M Harrison, ${ }^{6,7}$ Kate L Thomson, ${ }^{8,9}$ Helen Sage, ${ }^{8}$ Alexander Y Ing, ${ }^{6,7}$ Paul J R Barton, ${ }^{1,2}$ Stuart A Cook, ${ }^{1,2,10}$ Daniel G MacArthur, ${ }^{4,5,11}$ and James S Ware ${ }^{1,2,3,5 *}$

${ }^{1}$ Cardiovascular Genetics and Genomics, National Heart and Lung Institute, Imperial College London, London, UK

${ }^{2}$ Cardiovascular Research Centre, Royal Brompton \& Harefield NHS Foundation Trust, London, UK

${ }^{3}$ MRC London Institute of Medical Sciences, Imperial College London, London, UK

${ }^{4}$ Analytic \& Translational Genetics Unit, Massachusetts General Hospital, Boston, Massachusetts, USA

${ }^{5}$ Program in Medical and Population Genetics, Broad Institute of MIT \& Harvard, Cambridge, Massachusetts, USA

${ }^{6}$ Laboratory for Molecular Medicine, Partners HealthCare Personalized Medicine, Cambridge, Massachusetts, USA

${ }^{7}$ Department of Pathology, Massachusetts General Hospital and Harvard Medical School, Boston, Massachusetts, USA

${ }^{8}$ Oxford Medical Genetics Laboratory, Oxford University Hospitals NHS Foundation Trust, Oxford, UK

${ }^{9}$ Division of Cardiovascular Medicine, Radcliffe Department of Medicine, University of Oxford, Oxford,UK

${ }^{10}$ National Heart Centre Singapore, Singapore, Singapore

${ }^{11}$ Department of Medicine, Harvard Medical School, Boston, Massachusetts, USA

${ }^{*}$ Correspondence should be addressed to Dr James Ware: j.ware@imperial.ac.uk 


\section{Main text}

Recent work by Shah and colleagues ${ }^{1}$ demonstrated that many variants in the ClinVar database $^{2}$ are misclassified, and that disease-specific allele frequency (AF) thresholds can identify wrongly classified alleles by flagging variants that are too prevalent in the population to be causative of rare penetrant disease. While we agree with the main conclusions of this work, the authors compare their AF filtering approach to our recently published method ${ }^{3}$, concluding that the method we advanced "may be prone to removing potentially pathogenic variants". This is incorrect. Here we demonstrate that our approach is robust, and further illustrate the power of disease-specific AF thresholds for investigating the genetic architecture of disease.

Both methods compare the population frequency of a variant with the prevalence of a disease. However, we advocate considering a fuller definition of disease architecture that explicitly incorporates penetrance and genetic heterogeneity. Using these parameters, we define the maximum AF at which a variant can be observed in the general population to be a credible candidate to cause a defined disease, under the specified genetic architecture. Importantly, we consider each ethnic sub-population separately ('popmax'), and account for sampling variance in reference datasets ${ }^{4}$. We previously demonstrated that our framework markedly improves signal:noise for identification of penetrant Mendelian variants, without loss of sensitivity ${ }^{3}$.

It is worth restating some important caveats of filtering variants using AF. We must be vigilant for population-specific founder variants, especially in populations where the disease architecture may be distinct, such as the Finnish and Ashkenazi Jewish populations in the Genome Aggregation Database (gnomAD). Also, we do not filter variants observed as singletons due to the stochastic nature of population sampling.

Shah et al. report that, in their hands, our method inappropriately filters 15 "high confidence" Pathogenic/Likely Pathogenic ClinVar variants across five cardiac phenotypes (dilated cardiomyopathy, hypertrophic cardiomyopathy, arrhythmogenic right ventricular cardiomyopathy, long QT syndrome (LQT), and Brugada syndrome). The work of Shah et al. cannot be directly replicated as their methods and reference dataset are not fully available. Therefore, we have assessed these 15 variants using the larger and more comprehensive gnomAD dataset. We calculated a maximum credible population AF for a variant causative of 
each disease (defined as in Table 1 of Whiffin et al. - see Supplementary Table 1) given a minimum penetrance of $50 \%$.

With proper application of our approach in this reference population, four (of 15) variants flagged by Shah et al. are not filtered (Table 1). We curated the remaining 11 variants according to contemporary ACMG/AMP guidelines ${ }^{5}$ using cardioclassifier.org ${ }^{6}$, ClinVar, and the published literature. Five did not reach a Pathogenic/Likely Pathogenic classification (Table 1; Supplementary Table 3).

The remaining six variants did have sufficient evidence to be classified as (Likely) Pathogenic. For four of the variants, where ethnicity specific case AFs were available, we estimated their penetrance by comparing the case AFs to gnomAD, as previously described ${ }^{7}$. The penetrance of these variants ranged from $1.1 \%$ to $12 \%$ (Table 1 ). Crucially, the upper confidence intervals of all six penetrance estimates are well below the pre-specified $50 \%$ threshold. In other words, our approach appropriately filters these variants as incompatible with the specified genetic architecture.

We extended our analysis to evaluate all Pathogenic/Likely Pathogenic ClinVar variants for these five cardiac phenotypes. Starting with the same ClinVar VCF (clinvar_20170905.vcf.gz), we annotated variants reported to cause the specified diseases with the tiering strategy outlined by Shah et al. ${ }^{1}$ To identify variants above the maximum credible AF for each disease, we used the highest filtering allele frequency across all gnomAD populations ("popmax") for each variant represented, as described previously ${ }^{3}$. These data, and the code to reproduce this analysis, are available for download from https://github.com/ImperialCardioGenetics/ResponseToShahEtAl.

47 additional variants, previously reported as Pathogenic/Likely Pathogenic, were flagged as "insufficiently rare" by this analysis. We reassessed the clinical interpretation using contemporary ACMG/AMP ${ }^{5}$ guidelines. $45 / 47$ (95.7\%) were classified as VUS (Table 2; Supplementary Table 2), and two were classified as (Likely) Pathogenic, but with low penetrance, clearly below our defined genetic architecture (Table 1).

Across both analyses, we identified eight (Likely) Pathogenic, low-penetrance variants (Table $2)$, two of which recapitulate a known mechanism of low-penetrance. These variants are reported as disease-causing for Jervell Lange-Nielsen syndrome (LQT \& deafness) in biallelic 
states, but have low-penetrance for dominant LQT in heterozygous relatives ${ }^{8}$. As these examples demonstrate, our AF filtering framework effectively discriminates alleles with lower penetrance that require tailored counselling.

Within the ACMG/AMP framework, frequency evidence favouring a benign interpretation (BS1) does not preclude a (Likely) Pathogenic classification overall. For example, a common lowpenetrance variant may be seen recurrently in cases, showing statistical enrichment in cases over controls (PS4). This contradictory evidence should trigger closer inspection and lead to consideration of a low-penetrance architecture. In other contexts, a more conservative lowpenetrance architecture may be specified from the outset.

In conclusion, we previously introduced a statistically-robust, disease-specific framework to leverage reference population AF for variant assessment. We show that this method does not remove true pathogenic variants, provided that they fall within the pre-defined genetic architecture. Although a specified architecture may lead to some low-penetrance variants being flagged with evidence in favour of benign status (BS1), this does not prevent them achieving an actionable ACMG/AMP classification in combination with other lines of evidence. Indeed, flagging this group of variants for in depth review enables more nuanced reporting and counselling around low-penetrance variation.

\section{Supplemental Data description}

Supplementary Data include three tables.

\section{Acknowledgments}

This work was supported by the Wellcome Trust (107469/Z/15/Z), the Medical Research Council (UK), the NIHR Biomedical Research Unit in Cardiovascular Disease at Royal Brompton \& Harefield NHS Foundation Trust and Imperial College London, the Fondation Leducq (11 CVD-01), a Health Innovation Challenge Fund award from the Wellcome Trust and Department of Health, UK (HICF-R6-373), and by the National Institute of Diabetes and Digestive and Kidney Diseases and the National Institute of General Medical Sciences, and the National Human Genome Research Institute of the NIH (awards U54DK105566, 
R01GM104371, and UM1HG008900). E.V.M. is supported by the National Institutes of Health under a Ruth L. Kirschstein National Research Service Award (NRSA) NIH Individual Predoctoral Fellowship (F31) (award Al122592-01A1). A.H.O.-L. is supported by National Institutes of Health under Ruth L. Kirschstein National Research Service Award 4T32GM007748.

This publication includes independent research commissioned by the Health Innovation Challenge Fund (HICF), a parallel funding partnership between the Department of Health and the Wellcome Trust. The views expressed in this work are those of the authors and not necessarily those of the Department of Health or the Wellcome Trust.

\section{Declaration of Interests}

The authors declare no competing interests.

\section{Web Resources}

Github repository: https://github.com/ImperialCardioGenetics/ResponseToShahEtAl

\section{References}

1. Shah N., Hou Y-C.C., Yu H-C., Sainger R., Caskey C.T., Venter J.C. and Telenti A. (2018). Identification of Misclassified ClinVar Variants via Disease Population Prevalence. AJHG. 102(4), 609-619.

2. Landrum M.J., Lee J.M., Riley G.R., Jang W., Rubinstein W.S., Church D.M. and Maglott D.R. (2014). Nucleic Acids Res. Jan 1;42(1):D980-5.

3. Whiffin N., Minikel E., Walsh R., O'Donnell-Luria A.H., Karczewski K., Ing A.Y., Barton P.J.R., Funke B., Cook S.A., MacArthur D. and Ware J.S. (2017). Using high-resolution variant frequencies to empower clinical genome interpretation. Genet. Med. Oct; 19(10):1151-1158.

4. Lek M., Karczewski K.J., Minikel E.V., Samocha K.E., Banks E., Fennell T., O'DonnellLuria A.H., Ware J.S., Hill A.J., Cummings B.B. et al. (2016) Analysis of protein-coding genetic variation in 60,706 humans. Nature. Aug 18;536(7616):285-91. 
5. Richards S., Aziz N., Bale S., Bick D., Das S., Gastier-Foster J., Grody W.W., Hegde M., Lyon E., Spector E. et al. (2015). Standards and guidelines for the interpretation of sequence variants: a joint consensus recommendation of the American College of Medical Genetics and Genomics and the Association for Molecular Pathology. Genet. Med. May;17(5):405-24.

6. Whiffin N., Walsh R., Govind R., Edwards M., Ahmad M., Zhang X., Tayal U., Buchan R., Midwinter W., Wilk A.E. et al. (2018). CardioClassifier: disease- and gene-specific computational decision support for clinical genome interpretation. Genet. Med. Jan 25 [Epub ahead of print].

7. Minikel E.V., Vallabh S.M., Lek M., Estrada K., Samocha K.E., Sathirapongsasuti J.F., McLean C.Y., Tung J.Y., Yu L.P., Gambetti P. et al. (2016). Quantifying prion disease penetrance using large population control cohorts. Sci. Transl. Med. Jan 20;8(322):322ra9.

8. Tranebjaerg L., Bathen J., Tyson J., and Bitner-Glindzicz M. (1999). Jervell and Lange-Nielson syndrome: a Norwegian perspective. Am. J. Med. Genet. Sep 24;89(3):13746. 
Tables

Table 1: Curations and penetrance estimates of 15 variants flagged by Shah et al. and 2 additional candidate lowpenetrance variants identified in this analysis. Full details can be found in Supplementary Table 3. In the Shah et al analysis 15 variants with "high-confidence" pathogenic assertions in ClinVar were reported to exceed the maximum credible population allele frequency for a pathogenic variant defined by our framework. In our reanalysis, four of these are not filtered by our recommended application of this approach (using gnomAD reference populations), five do not have sufficient evidence for a pathogenic assertion, and six are likely pathogenic but with low penetrance. 


\begin{tabular}{|c|c|c|c|c|c|c|}
\hline Phenotype & Gene & Cdna & Penetrance & ACMG evidence & ACMG class & conclusion \\
\hline $\mathrm{HCM}$ & MYBPC3 & c. $3330+5 G>C$ & $12.0 \%(3.0-45.0 \%)$ & PVS1, PS4, PP1_strong & Pathogenic & $\begin{array}{l}\text { Likely pathogenic but with low } \\
\text { penetrance }\end{array}$ \\
\hline LQTS & KCNQ1 & c. $1588 \mathrm{C}>\mathrm{T}$ & $2.5 \%(0.64-9.8 \%)$ & PVS1, PS4_moderate & Likely Pathogenic & $\begin{array}{l}\text { Likely pathogenic but with low } \\
\text { penetrance }\end{array}$ \\
\hline LQTS & KCNQ1 & c. $1781 \mathrm{G}>\mathrm{A}$ & $4.1 \%(1.0-16 \%)$ & $\begin{array}{l}\text { PS4, PM1, PP1, } \\
\text { PS3_supporting }\end{array}$ & Likely Pathogenic & $\begin{array}{l}\text { Likely pathogenic but with low } \\
\text { penetrance }\end{array}$ \\
\hline Brugada & SCN5A & c. $3956 \mathrm{G}>\mathrm{T}$ & $1.1 \%(0.010-12 \%)$ & PS3, PP2, PP3, PP1 & Likely Pathogenic & $\begin{array}{l}\text { Likely pathogenic but with low } \\
\text { penetrance }\end{array}$ \\
\hline Brugada & SCN5A & c.5129C>T & $\begin{array}{l}\text { Ethnicity matched case } \\
\text { data not available }\end{array}$ & PS3, PM1, PP3 & Likely Pathogenic & $\begin{array}{l}\text { Likely pathogenic but with low } \\
\text { penetrance }\end{array}$ \\
\hline $\begin{array}{l}\text { Brugada/ } \\
\text { LQTS }\end{array}$ & SCN5A & c. $1099 \mathrm{C}>\mathrm{T}$ & $\begin{array}{l}\text { Ethnicity matched case } \\
\text { data not available }\end{array}$ & PS3, PM1, PP3 & Likely Pathogenic & $\begin{array}{l}\text { Likely pathogenic but with low } \\
\text { penetrance }\end{array}$ \\
\hline LQTS & SCN5A & c. $4877 \mathrm{G}>\mathrm{A}$ & - & PS3_moderate, PP2, PP3 & VUS & $\begin{array}{l}\text { Insufficient evidence for a pathogenic } \\
\text { assertion }\end{array}$ \\
\hline LQTS & $K C N Q 1$ & c.364dupT & - & PVS1 & VUS & $\begin{array}{l}\text { Insufficient evidence for a pathogenic } \\
\text { assertion }\end{array}$ \\
\hline $\mathrm{HCM}$ & MYL3 & c. $170 \mathrm{C}>\mathrm{G}$ & - & $\begin{array}{l}\text { PS3_moderate, } \\
\text { PP1_moderate, PP2 }\end{array}$ & VUS & $\begin{array}{l}\text { Insufficient evidence for a pathogenic } \\
\text { assertion }\end{array}$ \\
\hline LQTS & SCN5A & c. $5872 \mathrm{C}>\mathrm{T}$ & - & PVS1 & VUS & $\begin{array}{l}\text { Insufficient evidence for a pathogenic } \\
\text { assertion }\end{array}$ \\
\hline LQTS & KCNQ1 & c. $1085 A>G$ & - & PM1, PP3 & VUS & $\begin{array}{l}\text { Insufficient evidence for a pathogenic } \\
\text { assertion }\end{array}$ \\
\hline DCM & LMNA & c. $961 \mathrm{C}>\mathrm{T}$ & - & $\begin{array}{l}\text { PVS1, PP1_Strong, PM2, } \\
\text { PS4_Moderate, } \\
\text { PS3_Moderate }\end{array}$ & Pathogenic & Not filtered using gnomAD \\
\hline Brugada & SCN5A & c. $4885 \mathrm{C}>\mathrm{T}$ & - & PVS1, PS3_moderate & Likely Pathogenic & Not filtered using gnomAD \\
\hline LQTS & $K C N Q 1$ & $\begin{array}{l}\text { c.573_577delG } \\
\text { CGCT }\end{array}$ & - & $\begin{array}{l}\text { PVS1, PP1_supporting, } \\
\text { PS3_supporting }\end{array}$ & Likely Pathogenic & Not filtered using gnomAD \\
\hline $\mathrm{HCM}$ & MYBPC3 & c. $3181 \mathrm{C}>\mathrm{T}$ & - & PVS1, PP1_strong & Pathogenic & Not filtered using gnomAD \\
\hline LQTS & KCNE1 & c. $226 \mathrm{G}>\mathrm{A}$ & $1.7 \%(0.58-5.2 \%)$ & PS4, PS3 & Pathogenic & $\begin{array}{l}\text { Likely pathogenic but with low } \\
\text { penetrance* }\end{array}$ \\
\hline LQTS & $K C N Q 1$ & c. $1664 \mathrm{G}>\mathrm{A}$ & $1.6 \%(0.22-12 \%)$ & $\begin{array}{l}\text { PM5, PM1, } \\
\text { PS3_moderate, PP3 }\end{array}$ & Likely Pathogenic & $\begin{array}{l}\text { Likely pathogenic but with low } \\
\text { penetrance }^{*}\end{array}$ \\
\hline
\end{tabular}

*Variants identified by wider gnomAD analysis 
Table 2: The final classifications of 15 variants flagged by Shah et al. and 47 additional variants identified in this analysis.

\begin{tabular}{|l|l|l|l|l|}
\hline Variant class & Total variants & $\begin{array}{l}\text { Insufficient evidence for } \\
\text { a pathogenic assertion }\end{array}$ & $\begin{array}{l}\text { Likely pathogenic but } \\
\text { with low penetrance }\end{array}$ & Not filtered using gnomAD* \\
\hline $\begin{array}{l}\text { Flagged by Shah et } \\
\text { al. }\end{array}$ & 15 & $5(33.3 \%)$ & $6(40.0 \%)$ & $4(26.7 \%)$ \\
\hline Other set 1 & 10 & $8(80.0 \%)$ & $2(20.0 \%)$ & - \\
\hline Set 2 & 26 & $26(100.0 \%)$ & $0(0.0 \%)$ & - \\
\hline Set 3 & 11 & $11(100.0 \%)$ & $0(0.0 \%)$ & - \\
\hline & $\mathbf{6 2}$ & $\mathbf{5 0 ( 8 0 . 6 \% )}$ & $\mathbf{8 ( 1 2 . 9 \% )}$ & $\mathbf{4 ( 6 . 5 \% )}$ \\
\hline
\end{tabular}

*Either there is additional data in the unpublished cohort used by Shah et al that was not obtainable for this work, or the framework was applied incorrectly, for example by filtering singleton variants or known founder alleles 